to which trials of these interventions consider inequalities. We conducted a systematic review synthesising evidence on how different measures of inequality impact the uptake of, adherence to and effectiveness of behavioural weight management interventions in adults with overweight and obesity.

Methods We updated a systematic review from the US Preventive Services Taskforce to identify (cluster-) randomised controlled trials of primary care applicable behavioural weight management interventions in adults with overweight and obesity published prior to 5th March 2020. Two investigators independently screened articles for eligibility and conducted risk of bias assessment. We curated publication families for each trial. The PROGRESS-Plus framework (place of residence, race/ethnicity, occupation, gender, religion, education, socioeconomic status, social capital, plus other discriminating factors) was used to consider a comprehensive range of health inequalities. Data on trial uptake, intervention adherence or attendance, and weight change outcomes by PROGRESS-Plus criteria were extracted. Data were synthesised narratively, and Harvest plots were produced to summarise the impact of each criterion on uptake, adherence, and effectiveness.

Results One hundred and three studies (89 from previous review; 14 from updated search) were identified. The majority $(n=91)$ are trials of behavioural weight loss interventions; 12 are trials of behavioural weight maintenance interventions. At baseline, all studies reported age $(n=103)$ and $101 \quad(98 \%)$ reported gender/sex; $67(65 \%)$ studies reported race/ethnicity and $57(55 \%)$ education. The least reported criteria were place of residence $(n=3,2 \%)$ and religion $(n=1,1 \%)$. Ten studies (10\%) examined the impact of at least one PROGRESS-Plus criteria on uptake, $10(10 \%)$ on intervention adherence or attendance, $31(30 \%)$ on trial attrition, and 30 (29\%) on weight change. Due to heterogeneity in intervention type and measures used to assess PROGRESS-Plus criteria, a metaanalysis was not conducted. Further results will be synthesised by August 2021, including Harvest plots summarising inequalities at each stage by different PROGRESS-Plus criteria.

Conclusion We identified a lack of consideration of inequalities in trials of behavioural weight management interventions for adults, especially in relation to trial uptake and adherence, and substantial heterogeneity in measures used to assess PROGRESS-Plus criteria. Current evidence does not align with public health policy which prioritises health inequalities. Researchers should consider health inequities in the design, conduct and targeting of interventions to best inform policy decisions and practice.

\section{P42 WHAT IS THE EVIDENCE BASE SURROUNDING PARENTAL PHYSICAL ACTIVITY? A SYSTEMATIC SCOPING REVIEW OF THE LITERATURE}

${ }^{1}$ Rachel Simpson*, ${ }^{1}$ Kate Ellis, ${ }^{1}{ }^{2}$ Kathryn Hesketh, 'Esther van Sluijs. ${ }^{1}$ UKCRC Centre for Diet and Activity Research (CEDAR), MRC Epidemiology Unit, University of Cambridge, Cambridge, UK; ${ }^{2}$ UCL Great Ormond Street Institute of Child Health, University College London, London, UK

\subsection{6/jech-2021-SSMabstracts. 130}

Background Despite the known benefits of physical activity (PA) to physical and mental health, many people fail to achieve recommended PA levels. Given parents are less active than non-parent contemporaries, they constitute a large potential intervention population. Interventions should be based upon in-depth understanding of the target behaviour and its determinants. This scoping review (based on Arksey and O'Malley's guidelines (2005)) therefore aimed to provide an overview of the current evidence base for parental PA.

Methods Four databases (Medline, Embase, PsychInfo, Scopus) were systematically searched to identify peer-reviewed articles focusing on parental PA from 2005 onwards, including interventional, observational or qualitative study designs. Title and abstract screening was followed by duplicate full-text screening. Data extracted for all articles $(100 \%$ checked by a second reviewer) included study design, proportion of fathers in sample, and ages of children. For quantitative studies, PA assessment method and factors examined based on the SocioEcological Model were extracted, as were intervention target and approach for interventional studies, and questions addressed in qualitative studies. Narrative methods, tabulations and graphs were used to summarise results.

Results Of 14,913 unique records retrieved, 213 articles were included; 27 reported on multiple study designs. 173 articles reported on quantitative data (81 cross-sectional, 26 longitudinal, 76 interventional) and 58 qualitative. The majority of articles originated from North America (62\%); 53\% included only mothers, whilst $2 \%$ included only fathers. Articles most frequently represented parents of infants (55\% of articles), toddlers $(51 \%)$, preschoolers $(50 \%)$, and primary-school aged children (49\%). Parents of young and older adolescents were only represented in $28 \%$ and $18 \%$ of the articles respectively. The majority of quantitative articles only included selfreported PA (69\%). Observational articles focused on individual correlates/determinants $(90 \%)$ and to a lesser extent on interpersonal and environmental factors $(27 \%$ and $25 \%$ respectively). The majority of interventional articles related to full trials (71\%), rather than pilot or feasibility studies, and involved parents alone (59\%). Qualitative articles predominantly obtained information from focus groups or group interviews $(47 \%)$ or individual interviews (45\%), and most explored PA barriers and facilitators (57\%).

Conclusion A range of quantitative and qualitative research has been conducted on parental PA. This review highlights areas for conducting systematic reviews of related articles, such as those focused on the PA of parents of specific groups of children. It also identifies gaps in the literature, for example around paternal PA, to inform intervention development.

\section{P43 A SYSTEMATIC REVIEW OF THE EFFECTIVENESS OF PERSONAL LETTERS TO HEALTHCARE PROFESSIONALS IN CHANGING PROFESSIONAL BEHAVIOURS}

${ }^{1,2}$ Aikaterini Grimani* ${ }^{1,3}$ Mei Yee Tang, ${ }^{1,3}$ Louis Goffe, ${ }^{1,3}$ Fiona Beyer, ${ }^{1,3}$ Falko Sniehotta,
${ }^{1,2}$ Ivo Vlaev. ${ }^{1}$ NIHR Policy Research Unit in Behavioural Science, Newcastle University,
Newcastle, UK; ${ }^{2}$ Warwick Business School, University of Warwick, Coventry, UK;
${ }^{3}$ Population Health Sciences Institute, Newcastle University, Newcastle, UK

10.1136/jech-2021-SSMabstracts. 131

Background Letters are regularly sent by healthcare organisations to healthcare professionals to encourage them to take action, change practice or implement guidance (e.g., regarding immunisation, blood pressure measurement, prescription, referral). However, whether letters are an effective tool in delivering a change in healthcare professional behaviour is currently uncertain. A systematic review was conducted to identify what information and behaviour change techniques (BCTs) within letters have the potential to optimise behaviour change in healthcare professionals. 
Methods A comprehensive literature search of published and unpublished studies (grey literature) in five electronic databases (MEDLINE, Embase, PsycINFO, the Cochrane Library and CINAHL) was conducted to identify randomized controlled trials (RCTs) that met the inclusion criteria. Supplementary searches in Google Scholar, hand searches in relevant journals, and backward and forward citation searching of included studies and relevant reviews were also conducted. A systematic approach to searching, screening, reviewing and data extraction was applied in accordance with the process recommended by the Cochrane Collaboration. Two researchers examined titles, abstracts, full-texts for eligibility independently. Risk of bias (RoB) was assessed using Cochrane RoB for randomized controlled trials. In addition, intervention and comparator group material were coded by two independent reviewers into their BCTs using the BCT taxonomy version 1. Disagreements were resolved by a consensus procedure.

Results The search of the predefined databases resulted in 6004 records, while a further 1334 records were found from other sources, giving a total of 7,338 citations. After duplicates were removed $(\mathrm{n}=3,241)$, a total of 4,097 citations were screened against the inclusion criteria. Of these, 4,012 citations were excluded on the basis of title, keywords, and abstract. The full texts of the remaining articles $(n=85)$ were then assessed against the inclusion criteria, resulting in 36 RCTs being retained. The studies were assessed as either low or unclear risk of bias. The majority of the RCTs investigated behaviour regarding antibiotic prescribing among physicians. The findings suggest that letters that incorporate social norms techniques are effective and can lead to behaviour change, with the potential for change in clinical outcomes. The effects might be enhanced by use of behavioural instruction, and a high-profile messenger.

Conclusion The evidence from this review provides important insights for healthcare providers and health authorities regarding how to formulate the communication, and what information and behaviour change techniques to include in order to optimise the potential effect on the behaviour of the receivers.

\section{P44 RISK-AWARENESS, ATTITUDE AND PRACTICE OF HEALTH CARE PROFESSIONALSTOWARDS REDUCING TOBACCO SMOKE EXPOSURE AMONG PREGNANT WOMEN AND CHILDREN}

\begin{abstract}
1,2ZZinab Hassanein*, 1,3 Ilze Bogdanovica, 1,3 Tessa Langley, 1,3 Rachael Murray,
1,3,4 Jo Leonardi-Bee. 'Division of Epidemiology and Public Health, University of Nottingham, Nottingham, UK; ${ }^{2}$ Public Health department, Assiut University, Assiut, Egypt; ${ }^{3}$ UK Centre for Tobacco and Alcohol Studies, University of Nottingham, Nottingham, UK; ${ }^{4}$ The Nottingham Centre for Evidence-Based Healthcare: JBI Institute, University of Nottingham, Nottingham, UK
\end{abstract}

\subsection{6/jech-2021-SSMabstracts. 132}

Background Approximately 50\% of Egyptian pregnant nonsmoking women and $55 \%$ of children are exposed to secondhand smoke (SHS), representing a major public health concern. Health care professionals (HCPs) are well placed to help in preventing exposure and its associated health harms by increasing risk-awareness in these groups. This study aimed to explore risk-awareness, attitudes and practice of HCPs in maternal and child health $(\mathrm{MCH})$ clinics in Egypt towards SHS exposure among pregnant women and children.

Methods A survey was distributed to all HCPs working in public $\mathrm{MCH}$ clinics in primary and secondary health care centres in Assiut city, Egypt in 2020. The questionnaire included 12 questions exploring risk-awareness, 10 questions for attitude and 5 questions for counselling practice of HCPs. Scores on risk-awareness were classified as 'low', and 'high' risk awareness, while attitudes towards SHS exposure were classified as 'low', and 'high' negative attitudes. Scores on counselling practices were classified as good practice if $75 \%$ of questions answered with always or sometimes. Univariate analysis was performed to explore factors associated with high risk-awareness, high negative attitude and good counselling practice.

Results 367 HCPs participated in the survey (response rate 68.5\%): 45\% nurses, 21\% gynaecologists/obstetricians, 16\% paediatricians, $9 \%$ general practitioners and $9 \%$ midwives. $12.5 \%$ of HCPs reported being smokers and $70.3 \%$ reported exposure to SHS in their workplace and $51.7 \%$ in their homes. $22.1 \%$ of participants reported having received training on smoking cessation. $55.9 \%$ had high awareness of the risks of SHS exposure to health of pregnant women and children. General practitioners and paediatricians had higher risk awareness than other specialities $(\mathrm{P}<0.001)$. 52.9\% of HCPs had high negative attitude towards SHS exposure among pregnant women or children. This finding was more common among HCPs who were female HCPs $(57.4 \%, \mathrm{P}=0.01)$, working in rural areas $(61.3 \%, \mathrm{P}=0.02)$ and who reported not being exposed to SHS at home $(59.9 \%, \mathrm{P}=0.001) .51 .8 \%$ of HCPs reported having good counselling practice regarding SHS exposure. This was significantly associated with being female $(56.2 \%, P=0.01)$, serving a rural population $(68.5 \%$, $\mathrm{P}=0.001)$, receiving previous training on smoking cessation (72.8\%, $\mathrm{P}=0.001)$ and not being exposed to SHS at home $(61.6 \%, \mathrm{P}=0.001)$.

Conclusion Egyptian HCPs' awareness, attitude and practice in relation to the risks of SHS to pregnant women and children is inadequate. It is essential for them to receive additional education and training to enable them to help pregnant women/mothers of children to avoid SHS exposure.

\section{P45 THE WE CAN QUIT2 SMOKING CESSATION TRIAL KNOWLEDGE EXCHANGE AND DISSEMINATION: PRIORITIES FROM A COMMUNITY PERSPECTIVE, AND RECOMMENDATIONS FOR FUTURE RESEARCH AND POLICY}

${ }^{1}$ Stefania Castello* ${ }^{1}$ Catherine Darker, ${ }^{2} J$ oanne Vance, ${ }^{3}$ Elaine Buckle ${ }^{3}$ Caitriona Reynolds, ${ }^{3}$ Aine Buggy, ${ }^{2}$ Kevin O'Hagan, ${ }^{4}$ Norma Cronin, ${ }^{5}$ Nadine Dougall, ${ }^{6}$ Declan Devane. ${ }^{1}$ Public Health and Primary Care, Trinity College Dublin, Dublin, Ireland; ${ }^{2}$ Community Cancer Prevention, Irish Cancer Society, Dublin, Ireland; ${ }^{3}$ Tobacco Free Ireland Programme, Health Service Executive, Dublin, Ireland; ${ }^{4}$ National Women's Council, Dublin, Ireland; ${ }^{5}$ School of Health and Social Care, Edinburgh Napier University, Edinburgh, UKi ${ }^{6}$ Trials Methodology Research Network and School of Nursing and Midwifery, HRB Trials Methodology Research Network and NUI Galway, Galway, Ireland; 'Usher Institute, University of Edinburgh, Edinburgh, UK

\subsection{6/jech-2021-SSMabstracts. 133}

Background We Can Quit (WCQ) is a tailored communitybased smoking cessation intervention comprising group support delivered by trained lay women with optional access to combination Nicotine Replacement Therapy (NRT) without charge. It was designed for women living in socioeconomically disadvantaged areas in Ireland. A pilot cluster randomised controlled trial (WCQ2) determined that WCQ was feasible and highly acceptable to the target group. A key aim of the trial 